\title{
Strong Ties and Weak Ties Rationality: Theory and Scale Development
}

\author{
Louise Sundararajan ${ }^{1}\left[\right.$ [ $\cdot$ Kuang-Hui Yeh ${ }^{2}$ (])
}

Accepted: 30 August 2021 / Published online: 3 September 2021

(๑) The Author(s), under exclusive licence to Springer Science+Business Media, LLC, part of Springer Nature 2021

\begin{abstract}
Strong Ties and Weak Ties Rationality Scale (STWTRS) is a theory-driven questionnaire designed to capture cultural differences in reasoning about the world. It is intended to demonstrate empirically the heuristic value of the ontological turn that shifts the focus of cultural analysis from the down-stream values, beliefs, and behaviors to the upstream process of thinking and reasoning that is rooted in the local ways of being. This paper will present theory development, preliminary results, and potential contributions of this scale toward better understanding of the culturally different other.
\end{abstract}

Keywords Strong ties versus weak ties · Ontological turn · COVID-19 pandemic . Rationality · Culture

Strong Ties and Weak Ties Rationality Scale (STWTRS) is a theory-driven questionnaire designed to capture cultural differences in reasoning about the world. It offers an explanatory model of culture that can shed light on how people as agents reason about and interpret their worlds. The cultural insider's interpretative framework has not been the focus in conventional cultural theories. Neither self-construals, such as individualism vs collectivism, nor ecological factors such as high vs low mobility shed any light on how the cultural insiders reason about the world. In fact, taking the culturally different other's assumptions and truth claims seriously requires nothing short of an ontological turn (Heywood, 2017), which entails a paradigm shift in the scientist's approach to folk theory-from assimilation that forces the phenomena into the Procrustean bed of science to accommodation that makes it possible for science to discover, when confronted with folk theories, the limitations of its own explanatory framework (for more details, see Shweder, 1992).

Louise Sundararajan

louiselu@frontiernet.net

1 Rochester, USA

2 Academia Sinica, Taipei, Taiwan 
Capitalizing on the revisionist potential of the ontological turn as exemplified by Richard Shweder's (1992) work, Beal (2020) claims that the goal of psychology is not simply to predict the outcome but rather to understand the interpretative framework behind the outcome. More specifically, he argues that in order to get at people's interpretive framework, "the surest way is not to interrogate people's values but to look instead at their ontologies" (p. 281). This strategic focus on people's being in and thinking about their world constitutes the theoretical thrust behind STWTRS.

\section{Theory Development}

The theory behind STWTRS intends to ground investigation of cultural differences on two distinctly different ontologies - strong ties (ST) and weak ties (WT). Consistent with Lévi-Strauss's (1969/1949) work on structures of kinship, ST (strongties relationship with kin) and WT (weak-ties relationship with non-kin) are two ontological framings that may be considered foundational in the sense that engaging the other as kin or non-kin has far reaching ramifications for how we relate to and reason about the world. Guided by this intuition, we integrated insights from multiple disciplines to develop a theory of ST and WT rationalities.

The sociologist Granovetter (1973) was the first to call attention to the distinction between ST and WT-the former refers to networking with closely related others such as family and friends, whereas the latter, un-related others, such as acquaintances and strangers. To this network theory, psychology (Oishi, 2014; Oishi \& Kesebir, 2012) adds the ecological factor of mobility: ST networking is privileged in environments where there is little choice in relationships, due to low residential and relational mobility. By contrast, WT networking is associated with high mobility which allows people to make deals with un-related strangers, and to enter or exit a relationship at will. Within this framework of mobility and cross cultural psychology in general, the differences between ST and WT networks tend to fall along the collectivism versus individualism divide. In response to the call for an ontological turn, we move upstream to investigate rationality as the reasoning processes behind both ST/WT networking and collectivism/individualism.

Informed by Shweder (1991), Sundararajan (2020) defines rationality as "the assumptions of what is good, right, and beautiful, and the reasoning behind these assumptions" (p. 136). She further situates rationality in the context of ecological rationality (Todd et al., 2012) which in combination with evolutionary psychology suggest that our rationality and its ecological niche have coevolved in the evolutionary deep time. ST rationality prevailed in most of human history that goes as far back as the hunter gatherers. The ascendency of WT rationality is relatively recent. According to Schulz et al. (2019), systematic weakening of kinship-based relations (ST) in Western Europe can be traced back to the Pope's ban on cousin marriage in the Middle Ages.

These two elements- the ecological niches of ST versus WT, and their corresponding rationalities - come together in a third, ontological, dimension, opened up by Beal's (2020) notion of ontological framing. How we relate to entities or persons in the world are ontological frames, because it is through this particular 
relationship that we perceive the world in certain ways. Thus rationality and ontological framing are two sides of the same coin: ST and WT are the "ontological relationships" (p. 282) that lie at the root of our particular ways of reasoning referred to as rationality; and rationality in turn is the content of this intrinsically evaluative ontological frame of ST and WT.

More specifically, Sundararajan (2020) has proposed two different sets of logic behind ST or WT ontological framing that impact on how one reasons about the world. ST versus WT logic can be examined along the following dimensions: First, the difference in logic between ST and WT can be conceptualized in terms of two mental spaces: shared private space (inhabited by related, familiar others) versus public space (inhabited by unrelated others or total strangers). As an illustration, consider the bed-room as prototypical shared private space, where familiarity looms large, and no strangers are allowed; the market place, by contrast, is a prototypical public space where transactions among strangers are the norm.

Second, in a group context, whether the members are genetically connected or not will determine whether group survival takes precedence over individual survival, or whether self-sacrifice for the group makes sense or not. For instance, members of an extended family are genetically related such that helping to raise brother's son also helps to extend one's bloodline. This logic is the same as that of body cells each of which is ready to sacrifice itself for the greater good of the body. By contrast, in relationships where there is no genetic connection, for instance that between the virus and the host, self-sacrifice is less likely. Furthermore, kinship-based connection also determines the degree of mobility or choice one may have. For instance one cannot choose one's parents (strong ties), but one can choose to make or break relations with strangers (weak ties).

Lastly, corresponding to these differences in logic or reasoning are differences in cognitive style. The cognitive difference in experience-near versus experiencedistant, and concrete/perceptual versus abstract/conceptual modes of processing may have co-evolved with ST versus WT ancestral environment respectively. As Moffett (2018) points out, tracking intimate others (ST) and tracking unfamiliar others (WT) require different cognitive mechanisms. An anonymous group of strangers, for instance the market place, need markers to know who belongs to the group and who does not. Markers are abstract categories such as race, belief, religion, profession, nationality, and so on. The use of abstract categories to track others in WT environment necessarily capitalizes on experience-distant, abstract/ conceptual modes of processing. Intimate communities of ST, for instance among people who share the private space of a family or neighborhood, have no need for markers to determine group membership, since their group identity is based on the bloodline or a life shared in common for decades if not generations. Thus instead of abstract categories, intimate groups capitalize on the experience-near, concrete/perceptual mode of processing, such as facial expression, tone of voice, smell, and touch, to track the other. A related difference lies in holistic versus analytic thinking. Concrete, experiential approach to the world is conducive to holistic thinking that treats things as one whole piece of cloth, instead of breaking them down into disparate units of analysis characteristic of analytic thinking. 
Another difference is that holistic thinking focuses on the qualitative, whereas analytic thinking, the quantifiable aspect of things.

All of these cultural differences are well documented in the literature on collectivism versus individualism (Nisbett, 2003). For our purposes, these are placed under a different register, as differences in rationality between ST and WT ontological framing (see Table 1).

\section{Application to Culture Analysis}

For an illustration of the ST vs WT rationality, we cast Shweder et al.'s (1987) study of moral reasoning into the framework of Table 1. Shweder et al. (1987) wanted to know why in a temple town in East India, the cultural insiders considered it a sin for widows to eat fish. To investigate cross cultural moral reasoning in response to this question, Shweder and colleagues collected interviews of both Indian and US samples. The findings are summed up by Shweder as follows (cited in Beal, 2020, p. 282):

The ontological framing certainly produces dramatic differences in moral judgment but the content of the relevant frames (the positing of a status obligation associated with the station of widowhood and the continued mutual reliance of husband and wife even after death versus the obligation to protect the personal liberties and areas of discretionary choice of autonomous individuals) results in a personal attachment (associated with that status or station in life in this particular tradition) to values (for example, loyalty versus liberty) that seem very much like a part of a package deal for moral cognition (and motivation).

This dense summary of Shweder can be unpacked with Beal's (2020) proposal of a hierarchical organization of moral cognition: The ontological framing (a), such as "widow," constitutes the basis for the content of the frame (b) that is articulated as the evaluative, interpretative narratives of moral reasoning, such as "it is a sin to eat fish," which in turn gives rise to unique sensitivity or personal attachment to abstract values (c) such as loyalty or liberty. As exemplary of the ontological turn, Shweder's approach stands in sharp contrast to the conventional approach in psychology.

Why is it a sin for widows in a temple town in India to eat fish? The conventional answer from psychology would be: It is their beliefs, values, developmental history, social conditioning... the list goes on. All of these variables are downstream factors (c) in Beal's (2020) hierarchical organization of moral cognition. By contrast, Shweder et al. (1987) considered the upstream (a and b) ontological framing and its evaluative, interpretative narratives as primary motivators of human behavior. To demonstrate how, according to Shweder, ontological categories provide good reasons for doing the right thing, and how ontological assumptions have priority over downstream values and beliefs, we cast Shweder's summary above into the framework of Table 1.

a Ontological framing: ST vs WT framing of the widow 


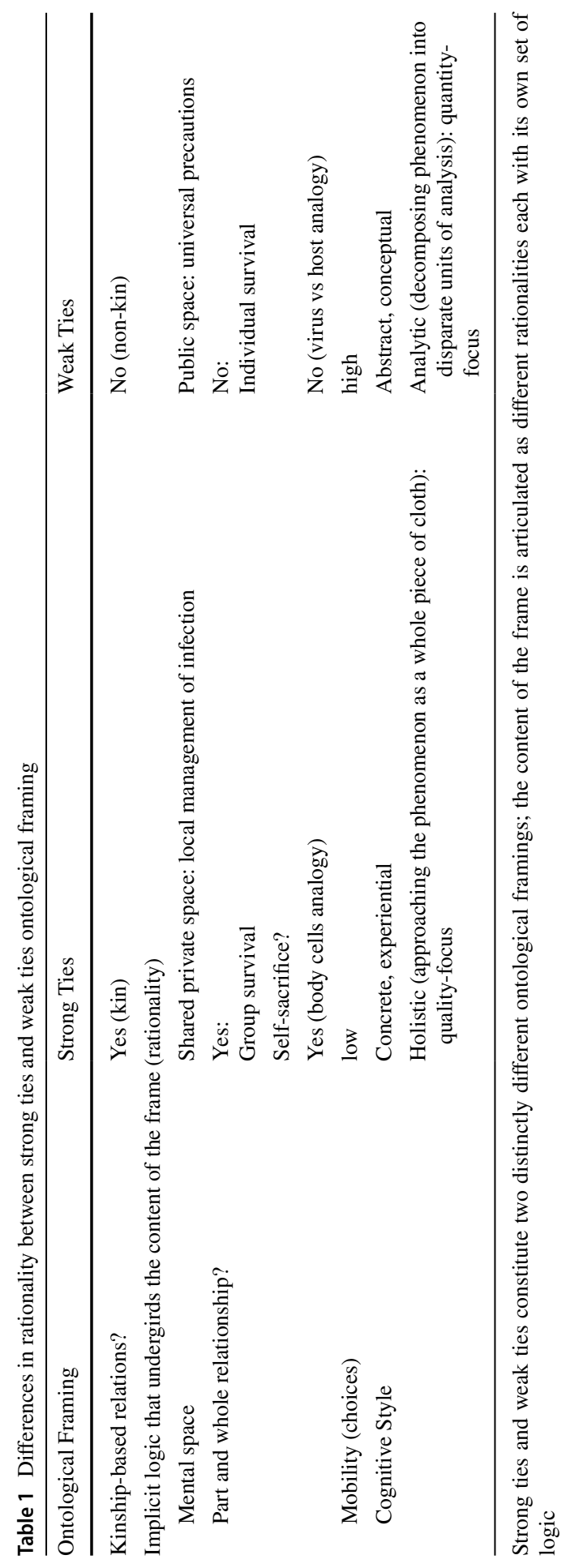


Indian sample: The widow is a member of the close-knit kinship-based group (i.e., the extended family).

US sample: Husband and wife's relationship is by contract which is annulled by death ("till death do we part" so goes the wedding vow).

b Content of the frame: Interpretative narratives generated by the framing. These narratives can be analyzed in terms of the following components of ST or WT logic/rationality (see Table 1):

1. Part and whole relationship:

Indian sample: In the kinship-based framework, the widow and her family are in a part and whole relationship, as evidenced by the continued mutual reliance of husband and wife even after death, such that what the widow eats may affect the soul of her deceased spouse.

US sample: In the contract-based relationship, the constituents function independent of each other such that what the widow eats is her own business.

2. Mobility:

Indian sample: A relationship bound by kinship ties has low mobility which may manifest as low degrees of freedom in choice making. For instance, one does not choose one's parents nor one's mate in some strong ties societies. The low mobility/choice is also manifest in the extensive codifications of one's roles, status, and obligations within the web of intimate relations.

US sample: Contract-based relationships have high degrees of mobility/choice, such that widowhood does not define one's position in life or society.

3. Cognitive styles: holistic versus analytic

Indian sample: The quality-focus of holistic thinking is manifest in the widow's dietary concerns. Foods are divided into two broad categories, hot or cold, based on their quality (conducive to sexual desires or not), not quantity (calorie count). Fish along with garlic and onions are hot foods that increase sexual desires thereby undermining the ideals of widowhood in which the woman is supposed to remain faithful to her deceased spouse.

US sample: Analytic thinking would support diet to reduce calorie intake, but not the web of associations that connect food restrictions (avoid hot food) with the right kind of behavior (avoid sex and sexual desires) suitable for one's specific position in life (widowhood).

\section{c Beliefs and values:}

Indian sample: loyalty

US sample: liberty.

This brief analysis reiterates the hierarchical organization of moral reasoning as suggested by Beal (2020): The ST or WT ontological framing (a) of widowhood constitutes the basis for the content of the frame $(\boldsymbol{b})$ that is articulated as the evaluative, interpretative narratives that differ across cultures in multiple dimensions (part/ whole, mobility, and holistic/analytic), which in turn give rise to unique, cultural specific sensitivity or personal attachment to abstract values (c) such as loyalty or liberty. Thus in contrast to cultural and cross cultural psychologies that focus on downstream beliefs and values (c) - such as collectivism versus individualism with their corresponding values of loyalty versus liberty, Beal (2020) and Shweder give 
priority to the ontological assumptions of the cultural insider, for whom it is the upstream reasoning that deepens one's sensitivity or attachments to certain cultural/ moral values/beliefs, and not the other way around.

This constitutes a paradigm shift from outcome (what one believes) to process (how one thinks) in moral cognition. Which form of questioning would be more predictive of people's judgments: framing questions or values questions? Beal (2020) asks:

For instance, participants could rate on a Likert scale their level of agreement with ontological framing statements such as "Fetuses are persons" or "Climate change is caused by humans" and also with values statements such as "I value personal liberty" or "I value personal responsibility." (p. 282)

And he places his bet on the framing questions. STWTRS is developed to test this assertion.

\section{Scale Construction}

To situate the reasoning/interpretative discourse of rationality in a universal context, we cast our scale development in the framework of the COVID-19 pandemic. More specifically, STWTRS consists of 20 evaluative statements concerning the COVID19 pandemic. These statements were constructed to encode the sets of ST versus WT logic as identified in Table 1. One example of item construction shall suffice.

The Chinese sociologist Fei (1992) sums up the major difference in group dynamics between traditional China (a strong ties society) and the West (a weak ties society) in the following terms: "Western society is represented by straws collected to form haystack, and Chinese society is represented by the ripples flowing out from the splash of a rock thrown into water" (p. 20). Cast into the framework of Table 1, Fei's analogies can be translated into the following sets of contrast: Characteristic of holistic thinking is the analogy of a part and whole relationship in terms of ripples that cannot be separated from one another; Characteristic of analytic thinking is the analogy of a bundle of straws each of which can be freely pulled without affecting the rest of the haystack.

To continue this contrast in thinking styles, we constructed two items for STWTRS. Corresponding to the straw analogy of weak ties is the following item:

An analogy of transmission of the coronavirus is match sticks: If one match stick is burning, the next match stick will catch fire if it is too close to it, but if the two match sticks are far apart, the second one will not catch fire.

Similar to the straws, the match sticks have no intrinsic connection to one another, a relationship characteristic of weak ties, according to Table 1. We deem these two statements, the straws and the match sticks, isomorphic because they share the same logic.

Based on the principle of isomorphism, we constructed a statement about the pandemic to match the ripples analogy of strong ties: 
Infectious decease works like contamination: One mouse dropping in the soup, and you have to throw out the whole pot of soup; one person gets infected, and the whole community is endangered.

The notion of contagion and the analogy of the spreading ripples share the same logic of holistic thinking that supports the perception of a part and whole relationship.

Whereas the match-stick is a widely known analogy during the pandemic, the contagion analogy is our concoction. This is an example of how we constructed contrasting statements of rationality in our scale development: Using one readymade and widely used statement about the pandemic, we derived from it its logical antithesis, with the help of Table 1, to be encoded in another, newly minted statement about the pandemic. A scale of 20 items was developed in this way. Note that these items are narratives of reasoning about the world, which are different from the garden variety of abstract beliefs and values (such as loyalty or justice) in conventional measures of culture.

\section{Hypothesis Testing}

Based on the foregoing analysis, our hypothesis is twofold: First, in reasoning about the world, for instance about the pandemic, people follow a set of logic (see Table 1) that stems from their ontological framing of ST or WT, which can be articulated as evaluative statements about the world (the pandemic). Second, these upstream reasoning processes may have their distinct correlates in beliefs and values downstream.

To test this hypothesis, participants are asked to rate on a Likert scale 20 evaluative statements about the pandemic, with 10 items each that articulates the ontological framings of ST and WT, respectively. The task of rating the items is intended to activate the reasoning process, which is predicted to be able to differentiate between the two sets of logic-ST versus WT-that is encoded in the items. This leads to one testable prediction as follows:

H1: The participants can differentiate the STWTRS items in accordance with their implicit ST and WT logic/rationality.

Due to the wide margins of overlap and affinity (Sundararajan, 2020) between the measures of collectivism/ individualism and ST/WT, beliefs and values of collectivism/ individualism may be considered downstream correlates of ST/WT ontological priming. From this premise flows one testable prediction:

H2: ST versus WT ontological framings, as encoded in the STWTRS items, have correlates in beliefs and values that are conventionally associated with collectivism versus individualism, respectively, such as loyalty/obligation versus justice/ fairness in morality; and belief in just world versus belief in unjust world. 


\section{A Preliminary Study}

STWTRS was normed on a representative sample in Taiwan $(n=289)$. Outcome measures are the following well established measures of culture: Belief in a just world (BJW) (Dalbert et al., 2001), Moral Foundations Questionnaire (MFQ) (Graham et al., 2011), Tight and Loose Cultures (Gelfand et al., 2011), Hofstede Individualism index (Hofstede et al., 2008), and Dual Filial Piety Scale (Bedford \& Yeh, 2020; Yeh \& Bedford, 2003).

\section{Results and Discussion}

H1: The participants can differentiate the STWTRS items in accordance with their implicit ST and WT logic/rationality.

This hypothesis is partially confirmed, suggesting that participants could differentiate half of the items along the ST and WT divide. Exploratory factor analysis revealed two factors with items matching their theoretical construct: Factor one was labeled ST, because it had 5 items (loading from 0.85 to 0.60 ) that were constructed to articulate the ST logic/rationality of group survival, low mobility, and holistic thinking. Factor two was labeled WT, because it had 5 items (loading from 0.76 to 0.35 ) that were constructed to articulate the WT logic/ rationality of individual survival, high mobility, and analytic thinking. See Table 2

Consistent with the construct of ST and WT rationality, the importance of selfsacrifice in lockdown (item 13) has positive and high loading (0.85) on ST, and negative loading (-0.17) on WT. The other way around was true for the analytical thinking that considers a balance of interest between individual and group during lockdown (item 14), which had higher loading on WT (0.51), than on ST (0.15). Similarly, the idea of self-expression during lockdown (item 19) had negative loading on ST (-0.03), but positive and moderate loading on the WT (0.52), whereas the idea that complaining during lockdown may have negative impact on others (item 20) had high loading on ST (0.63), and low loading on WT (0.09). It is also interesting to note that the idea that rigorous lockdown is necessary to flatten the curve (item 9) had positive and high loading (0.79) on ST, but negative loading on WT (-0.20). By contrast, the universal precaution of mask, social distancing, and hand washing (item 11) had negative loading (-0.11) on ST, but positive and high loading (0.65) on WT.

H2: ST versus WT ontological framings, as encoded in the STWTRS items, have correlates in beliefs and values downstream that are conventionally associated with collectivism and individualism.

Correlations of ST and WT factors with values and beliefs are largely as expected to fall along the divide between collectivism and individualism: On 
Table 2 Exploratory factor analysis of STWTRS

\begin{tabular}{|c|c|c|}
\hline \multirow[t]{2}{*}{ STWTRS items } & \multicolumn{2}{|c|}{ Factors } \\
\hline & ST & WT \\
\hline $\begin{array}{l}\text { 13. Given that a lockdown is the most effective means of prevention, a spirit of self-sacri- } \\
\text { fice for the greater good is necessary to make it work }\end{array}$ & .845 & -.172 \\
\hline $\begin{array}{l}\text { 9. So long as people are allowed to move around freely, the infection cannot be contained. } \\
\text { That is why a rigorous lockdown is necessary to flatten the curve }\end{array}$ & .786 & -.204 \\
\hline $\begin{array}{l}\text { 20. It is important not to complain or gripe during a lockdown, because these words spread } \\
\text { negative energy and can hurt many people around you }\end{array}$ & .631 & .094 \\
\hline $\begin{array}{l}\text { 1. Infection works like contamination: one mouse dropping in the soup and you have to } \\
\text { throw out the whole pot. If one person gets infected, the whole community is endangered }\end{array}$ & .615 & .240 \\
\hline $\begin{array}{l}\text { 8. Not wearing a face mask during the pandemic is immoral just like littering or spitting, } \\
\text { and should be punished by law }\end{array}$ & .603 & .203 \\
\hline 15. Fact-checking is effective in debunking false information & .008 & .761 \\
\hline $\begin{array}{l}\text { 11. The key to successful prevention of personal infection lies in maintaining safe behav- } \\
\text { ior: hand washing, mask wearing, and social distancing }\end{array}$ & -.107 & .652 \\
\hline $\begin{array}{l}\text { 19. It is important to express yourself during a lockdown regardless of whether others } \\
\text { agree with you or not }\end{array}$ & -.029 & .515 \\
\hline $\begin{array}{l}\text { 14. In implementing a lockdown, it is important to balance the interests of the group with } \\
\text { the interests of individuals, so that one is not served at the expense of the other }\end{array}$ & .149 & .511 \\
\hline 18. It is Ok to be angry and to vent your frustration during a lockdown & .021 & .346 \\
\hline
\end{tabular}

Extraction method: Principle Axis. Rotation method: Promax

Bold values $=$ values of factor loading over .35

the Dual Filial Piety Scale, ST was highly related to Authoritarian filial piety $(r=0.59, p<0.000)$, and modestly related to Reciprocal filial piety $(r=.24$, $p<0.000)$, while WT was highly related to Reciprocal filial piety $(r=0.44$, $p<0.000$ ), but had no significant relationship with Authoritarian filial piety. With Individualism, ST was significantly and negatively correlated $(r=-0.16, p<0.01)$, while WT was significantly and positively correlated $(r=0.13, p<.05)$. On the Moral Foundations Questionnaire, ST, but not WT, was highly related to Ingroup bias $(r=.46, p<0.000)$; and ST $(r=0.55, p<0.000)$ relative to WT $(r=0.22$, $p<0.000)$ was more highly related to obedience to Authority. The two factors did not differ significantly in their correlations with Care. On BJW, ST was highly related $(r=0.42, p<0.000)$ to belief in a just world, but not to belief in an unjust world; the other way around was true with WT, which was significantly related to belief in an unjust world $(r=0.18, p<0.01)$, but not to belief in a just world.

\section{Results not Expected}

Correlation with Tight/Loose Cultures did not differentiate between the two factors. Likewise, On the Moral Foundations Questionnaire, correlations with Fairness and Purity failed to differentiate between the two factors. According to Sundararajan (2020), every culture has both ST and WT rationalities, such that it is the prevalence 
of ST or WT rationality that determines whether a society is ST or WT. In the case of the collectivistic or ST society of Taiwan, ST is the dominant while WT the nondominant rationality. Since rationality functions adaptively in its ecological niche, according to the theory of ecological rationality (Todd et al., 2012), it is reasonable to expect the non-dominant WT rationality to align itself with the dominant ST rationality in value judgments, when necessary. This pattern is also found in a larger scale $(\mathrm{n}=961)$ cross cultural study of STWTRS (Yeh et al., in revision), in which sharper differences between ST and WT were found across (collectivistic vs individualistic) than within (collectivistic or individualistic) societies.

\section{General Discussion}

The theory behind STWTRS posits that ST and WT are two distinct ontological framings each with its own set of logic that are articulated in our evaluative interpretations or explanations of the world. To test this hypothesis, a 20 items scale of reasoning about the pandemic was constructed and administered to a representative sample in Taiwan $(n=289)$. Exploratory factor analysis yielded two factors in which items loaded in predicted direction along the ST and WT divide. These two factors also exhibited correlations with measures of values and beliefs in cross cultural psychology in predicted direction to a large extent. This preliminary finding has far reaching implications for theory and measurement.

\section{Implications for Theory Development}

Why is it a sin for widows to eat fish in a temple down in East India? Contrary to the conventional explanations in terms of beliefs, behaviors, and values, the ontological turn, as demonstrated by Shweder et al.'s (1987) study, has a simpler and more elegant answer: Because they are widows. This approach suggests that there is a missing link in moral cognition, namely that values and beliefs per se do not have the power to motivate people (Beal, 2020), unless they make sense in the ontological universe of the cultural insiders. To shed some light on this missing link, the theory behind STWTRS posits that different ontological framings give rise to different ontological universes in which eating fish is (according to WT rationality) or is not (according to ST rationality) compatible with widowhood. Preliminary results of the Taiwan sample showed that just like widowhood, the same COVID-19 issues allow for different (ST vs WT) ontological framings that led to different correlates of values and beliefs downstream.

In a nutshell, the missing link in moral cognition lies in a conflation of "is" and "ought" in the ontological universe of the cultural insider: To the extent that to be a widow (is) entails how one can be a "good" widow (ought), the upstream ontological framing (is) has priority over downstream values and beliefs (ought). This has far reaching implications for the development of STWTRS. Different from the conventional measures in psychology, the items of STWTRS are not a list of de-contextualized statements that represent or capture the universal "essence" of certain 
downstream variables (personality traits, beliefs, values, behavioral tendency, etc.), so much as prompts that serve to activate the upstream reasoning process in a context-dependent manner-since understanding is always contextualized. In the final analysis, why should we invest so much time and energy on the peculiar diet restrictions of the widows in a temple down, a custom not generalizable to the rest of the population in India? The answer is that any type of mental phenomena is worth looking into if we want to expand the scope of our understanding of human beings as agents who need to construct a rational/logical universe in which they can do the right thing.

With understanding the cultural insider, rather than predicting behavior, as our objective, the preliminary results of STWTRS can shed some light on what makes sense in different cultures. High loadings on the ST factor are statements that emphasize the importance of self-sacrifice (item 13) and the need to reduce mobility by rigorous lockdown (item 9). Both items had low and negative loading on WT. Conversely, high loadings on the WT factor are statements that reiterate the importance of self-expression (item 19), and universal precautions based on quantifiable units of analysis such as social distancing, hand washing and mask wearing (item 11). Both items had low and negative loadings on ST. These findings suggest that to the rationality of ST framing, rigorous lockdown makes the most sense, whereas to that of WT framing, universal precaution makes the most sense. Lockdown reduces mobility far more effectively than procedures of universal precaution. To the extent that virus and information both need mobility to spread, lockdown and state censorship of information speak the same logic that favors low mobility. In WT rationality, preference for high mobility is also a double-edged sword: On the one hand, high relational mobility (free choice of partners) was found to be a significant predictor of the spread of COVID-19 during the first 30 days of country-wide outbreaks (Salvador et al., 2020); on the other hand, high mobility in information flow is necessary for the development of both science and democracy (Welzel, \& Inglehart, 2010).

These observations paint a very complex picture of cultural differences. To the extent that what appears to be meat to one rationality is poison to another, so to speak, we will not be able to deescalate the rising international tension in the post-pandemic era, unless we develop a keen interest in understanding the subjective mental world of cultural-insiders. To the extent that the framework of ST and WT makes it possible for us to see the sensibility of why widows should not eat fish, even if we are not from a temple town in India, the theory and measurement of STWTRS hold the potential to contribute to a more nuanced understanding of the culturally different other, an understanding that we are desperately in need of to prevent the racial and cultural bias from becoming another pandemic.

\section{Implications for Measurement}

The STWTRS has the following advantages over conventional cross cultural and cultural measures: 
1. Psychology has long been plagued by the serious limitations in cultural theories and measurement known as the WEIRD (Western, Educated, Industrialized, Rich, and Democratic) sampling problem (Henrich et al., 2010). WEIRD cultural traits associated with individualism have been well investigated and documented, but non-WEIRD traits associated with collectivism have been inadequately investigated and imprecisely measured. With its sensitivity to the ST rationality in non-WEIRD societies, STWTRS holds the potential to reverses the current trend in cross-cultural measures that are more predictive of WEIRD than non-WEIRD societies (Muthukrishna et al., 2020).

2. Data on the upstream reasoning process of ST versus WT rationality correlate well with the down-stream cultural differences in values and beliefs that are well documented in cross-cultural psychology. This suggests the possibility for STWTRS to integrate extant measures of culture into a more comprehensive framework that is theoretically defensible.

3. STWTRS is versatile in two respects: First, it is tailored according to the specific context, such that it is entirely possible to construct different items for a different context other than the pandemic, so long as there is isomorphism in logical structure among the measures, as determined by Table 1. Second, STWTRS is sensitive enough to detect differences within the same collectivistic (Taiwanese) culture in the present study. A larger scale cross cultural study (Yeh et al., in revision) also revealed different protocols in three Asian samples-Taiwanese, Chinese Yi minority, and Asian Americans.

4. STWTRS can extend itself to alternative testing. Previous research on ethnic minority groups (Ting \& Sundararajan, 2018) suggests that for populations not suitable for the paper and pencil tests, it is possible to manually code interview texts according to the matrix of rationality as presented in Table 1.

\section{Conclusion}

Strong Ties and Weak Ties Rationality Scale (STWTRS) is a theory-driven questionnaire to capture cultural differences in reasoning about the world. Preliminary results are promising. More important, it demonstrates empirically the heuristic value of the ontological turn (Heywood, 2017) that shifts the focus of cultural analysis from the down-stream values, beliefs, and behaviors to the upstream process of thinking and reasoning that is rooted in the local ways of being, otherwise known as ontological framing.

\section{References}

Beal, B. (2020). What are the irreducible basic elements of morality? A critique of the debate over monism and pluralism in moral psychology. Perspectives on Psychological Science, 15, 273-290.

Bedford, O., \& Yeh, K.-H. (2020). The contribution of Chinese process thought to psychology as a global science: Filial piety as an example. Review of General Psychology, 24, 99-109.

Dalbert, C., Lipkus, I. M., Sallay, H., \& Goch, I. (2001). A just and an unjust world: Structure and validity of different world beliefs. Personality and Individual Differences, 30, 561-577.

Fei, X. (1992). From the soil: The foundations of Chinese society (Gary G. Hamilton, \& Wang Zheng, Trans.). Oakland, CA: University of California Press. (Original work published 1947) 
Gelfand, M. J., Raver, J. L., Nishii, L., Leslie, L. M., Lun, J., Lim, B. C., et al. (2011). Differences between tight and loose cultures: A 33-nation study. Science, 332(6033), 1100-1104.

Graham, J., Nosek, B. A., Haidt, J., Iyer, R., Koleva, S., \& Ditto, P. H. (2011). Mapping the moral domain. Journal of Personality and Social Psychology, 101, 366-385.

Granovetter, M. S. (1973). The strength of weak ties. American Journal of Sociology, 78, 1360-1380.

Henrich, J., Heine, S. J., \& Norenzayan, A. (2010). The weirdest people in the world? Behavioral and Brain Sciences, 33, 61-83.

Heywood, P. (2017). Ontological Turn, The. In F. Stein, S. Lazar, M. Candea, H. Diemberger, J. Robbins, A. Sanchez, \& R. Stasch (Eds.), The Cambridge Encyclopedia of Anthropology. https://doi.org/10.29164/ 17ontology.

Hofstede, G., Hofstede, G. J., Minkov, M., \& Vinken, H. (2008). Values survey module 2008, from http:// www.geerthofstede.n1/vsm-08. Accessed 20 May 2019.

Lévi-Strauss, C. (1969/1949). The elementary structures of kinship (trans. J.H. Bell, J.R. von Stunner \& Needham). Boston: Beacon.

Moffett, M. W. (2018). The Human swarm. Basic Books.

Muthukrishna, M., Bell, A. V., Henrich, J., Curtin, C. M., Gedranovich, A., McInerney, J., \& Thue, B. (2020). Beyond western, educated, industrial, rich, and democratic (WEIRD) psychology: Measuring and mapping scales of cultural and psychological distance. Psychological Science, 31, 678-701.

Nisbett, R. E. (2003). The geography of thought. Free Press.

Oishi, S., \& Kesebir, S. (2012). Optimal social-networking strategy is a function of socioeconomic conditions. Psychological Science, 23, 1542-1548.

Oishi, S. (2014). Socioecological psychology. Annual Review of Psychology, 65, 581-609.

Salvador, C., Berg, M., Yu, Q., San Martin, A., \& Kitayama, S. (2020). Relational mobility predicts the increased speed of the spread of COVID-19: A 39-country study. Psychological Science, 31, 1236-1244.

Schulz, J. F., Bahrami-Rad, D., Beauchamp, J. P., \& Henrich, J. (2019). The Church, intensive kinship, and global psychological variation. Science, 366(6466), eaau5141.

Shweder, R. A. (1991). Thinking through culture: Expeditions in cultural psychology. Harvard University Press.

Shweder, R. A. (1992). Ghost busters in anthropology. In R.G., D’Andrade and Claudia Strauss (Eds.), Human motives and cultural models (pp. 45-57). Cambridge University Press.

Shweder, R., Mahapatra, M., \& Miller, J. (1987). Culture and moral development. In J. Kagan \& S. Lamb (Eds.), The emergence of morality in young children (pp. 1-83). University of Chicago Press.

Sundararajan, L. (2020). Strong-ties and weak-ties rationalities: Toward an expanded network theory. Review of General Psychology, 24, 134-143.

Ting, R. S-K., \& Sundararajan, L. (2018). Culture, cognition, and emotion in China's religious ethnic minorities: Voices of suffering among the Yi. Palgrave Studies in Indigenous Psychology Series. Springer Nature.

Todd, P. M., Gigerenzer, G., \& The ABC Research Group. (2012). Ecological rationality: Intelligence in the world. Oxford University Press.

Welzel, C., \& Inglehart, R. (2010). Agency, values, and well-being: A human development model. Social Indicators Research, 97, 43-63.

Yeh, K.-H., \& Bedford, O. (2003). A test of the dual filial piety model. Asian Journal of Social Psychology, 6, 215-228.

Yeh, K.-H., Sundararajan, L., Ting, R. K., Liu, C., Liu, T., \& Zhang, K. (in revision). Strong-ties and Weakties Rationalities: Toward an Ontological Turn in Cultural and Cross-Cultural Psychologies.

Publisher's Note Springer Nature remains neutral with regard to jurisdictional claims in published maps and institutional affiliations.

Louise Sundararajan received her Ph.D. in History of Religions from Harvard University, and her Ed.D. in Counseling Psychology from Boston University. She is editor-in-chief of Palgrave Studies in Indigenous Psychology. (https://www.palgrave.com/gp/series/15445?sap-outbound-id=). She has published extensively on topics related to culture and emotions, and is the author (2015) of Understanding emotion in Chinese culture: Thinking through psychology. https://www.springer.com/gp/book/9783319182209. 
Dr. Kuang-Hui Yeh is Research Fellow at the Institute of Ethnology, Academia Sinica, and Joint-Appointment Professor at Psychology Department, National Taiwan University. He is the author of "The Filial Piety of Chinese people: A psychological perspective"(2008) and "Cultivation of Chinese Personality: Perspective from the Context of Parent-child Interaction"(2017); and editor or co-editor of "Affect, Emotion, and Culture: Anthropological and Psychological Studies in Taiwanese Society"(2002), "Chinese Indigenous Psychology in Global Context: Reflections and Future Direction"(2013), "Asian Indigenous Psychologies in the Global Context"(2019), and "Global Psychology from Indigenous Perspectives: Visions Inspired by K. S. Yang"(2020). 